\title{
Comparison of Salivary and Serum Soluble CD44 Levels between Patients with Oral SCC and Healthy Controls
}

\author{
Seyedali Seyedmajidi ${ }^{1}$, Maryam Seyedmajidi ${ }^{1 *}$, Ramin Foroughi ${ }^{2}$, Amir \\ Zahedpasha $^{2}$, Zahra Zolfaghari Saravi ${ }^{3}$, Roghayeh Pourbagher ${ }^{4}$, Ali Bijani ${ }^{5}$, \\ Mina Motallebnejad ${ }^{6}$, Ali Monfared Shabestani ${ }^{7}$, Amrollah Mostafazadeh ${ }^{8}$
}

\begin{abstract}
Background: The most common type of oral cancer is oral squamous cell carcinoma. If it is diagnosed in the early stages; the success of the treatment can be increased. It seems that ELISA-based techniques as a screening tool for society are the most cost-effective methods for early diagnosis. CD44 is a key marker for the detection of SCC stem cells. The aim of this study was to compare the level of soluble CD44 in saliva and serum between patients with oral SCC and healthy controls. Materials and Methods: Saliva and serum were collected from 20 patients with primary OSCC and 20 healthy persons as control group. The samples were evaluated by an ELISA test kit. Data were analyzed by SPSS software version 22, chi-square, ANOVA, T-test and Spearman correlation test. Results: The mean of soluble CD44 level in serum and saliva of the patient and control groups are 531.51 \pm 228.95 and $453.3 \pm 113.74$ (for serum) and $48.53 \pm 59.02$ and $17.76 \pm 39.14$ (for saliva) respectively. There was no statistically significant difference in serum and saliva solCD44 level between the patient and control groups ( $\mathrm{P}$ value $=0.182$ and $\mathrm{P}$ value $=0.061$ respectively). Also, there was no significant correlation between the solCD44 level in each patient and control group in serum (P value $=0.61)$ and in saliva $(\mathrm{P}$ value $=0.445)$. Conclusions: Determination of solCD44 level in saliva and serum can be a useful method for diagnosis the person's involvement with cancer cells and the cancer in the early stages. But according to the controversial outcomes of past studies, larger and more accurate studies are needed in groups with more cases of oral cancer.
\end{abstract}

Keywords: CD44- oral squamous cell carcinoma- ELISA

Asian Pac J Cancer Prev, 19 (11), 3059-3063

\section{Introduction}

Oral cancer is the 11th most common malignancy in the world (Ghantous and Abu Elnaaj, 2017) and is the most common head and neck malignant tumors that has a high mortality rate. The most common type of oral cancer is Oral Squamous Cell Carcinoma (OSCC), which includes for approximately $90 \%$ of oral malignancies (Franzmann et al., 2005; Seyedmajidi et al., 2017; Seyedmajidi et al., 2018). An increased incidence of primary oral squamous cell carcinoma (OSCC) has been reported in young patients in Iran (Seyedmajidi et al., 2014; Seyedmajidi et al., 2018). Unlike other internal cancers, since the OSCC occurs in the oral cavity, is more visible and traceable, making it easier to take biopsy. However, despite remarkable advances in surgery, radiotherapy and chemotherapy, the mortality rate of people with oral cancers over a five year period, which is about $50 \%$, has not changed significantly since fifty years ago and this is maybe due to the fact that OSCC patients are diagnosed at late stages, and there is no reliable and definitive diagnostic marker for early diagnosis of this disease (Yang et al., 2010). Currently, OSCC is diagnosed usually in stages III and IV, with less effective treatment and a therapeutic success of $30 \%$. Also, the patients suffer from several complications after treatment of oral cancers (Motallebnejad et al., 2014).

It is estimated that if it is diagnosed in the early stages (I, II), the success of the treatment can be increased up to $80 \%$. Currently, OSCC is evaluated based on clinical examinations and histological analysis of suspected areas; however, it may not be detected in hidden areas $(\mathrm{Wu}$ et al., 2010). Accordingly OSCC is less detected in the early stages; early diagnosis by screening will be an obvious solution (Franzmann et al., 2005). Therefore, the use of sensitive and specific biomarkers for molecular detection along with histological sampling would be a convenient,

${ }^{1}$ Dental Materials Rresearch Center, ${ }^{4}$ Cellular and Molecular Research Center, ${ }^{5}$ Non-Communicable Pediatrics Diseases Research Center, Health Research Institute, ${ }^{2}$ Department of Oral and Maxillofacial Surgery, ${ }^{6}$ Department of Oral and Maxillofacial Diseases, School of Dentistry, ${ }^{3}$ Students Research Committee, ${ }^{7}$ Department of Medical Physics, School of Medicine, ${ }^{8}$ Department of Immunology, Babol University of Medical Sciences, Babol, Iran. *For Correspondence: ms_majidi79@yahoo.com 
accessible, non-invasive and inexpensive method for OSCC diagnosis (Yang et al., 2010, Wu et al., 2010). It seems that ELISA-based techniques as a screening tool for society are the most cost-effective methods; because its implementation is simpler and cheaper than DNA-based techniques (Franzmann et al., 2005). WHO has defined the conditions for the early detection of an illness: 1- The disease should be prevalent and have a serious relationship with mortality. 2- The prognosis of treatment should be improved in result of the screening. 3- The benefits of screening should be greater than the potential of its losses and costs. 4- The screening tests should definitely diagnose the disease in the early stages (Franzmann et al., 2005, Yang et al., 2010). Based on our knowledge about OSCC and the results of previous studies (Franzmann et al., 2005) and (Franzmann et al., 2007), it seems that the evaluation of CD44 in serum and saliva can be as a effective community screening tool for the early diagnosis of OSCC.

CD44 is a large group of transmembrane glycoproteins. The most common form is CD44s or standard. CD44 was first introduced by Dalchav as a molecule present in surface of T lymphocytes, granulocytes, and cortical thymocytes. This protein is known as an important factor in cell interactions and cell adhesion (Dalchau et al., 1980). Some isoforms, such as standard CD44, are present in normal cells, while other isoforms are expressed mostly in tumors. Normally, CD44 is attached to the hyaluronan to stabilize the association with the extracellular matrix. The tumor cells are linked to hyaluronic acid in the extracellular matrix via CD44. The reduction of CD44 is due to the fracture of the extracellular component (ectodomain), which occurs in a large number of malignant tumors. The interruption of CD44 cleaves the tumor cells from the extracellular matrix leads to the migration of them, causing the spread of tumors and metastasis (Takamune et al., 2007). It is believed that CD44 is a key marker for the detection of SCC stem cells. The presence of cancer stem cells was first described in 2007 by Prince et al. whom used CD44 to isolate cancer stem cells (Prince et al., 2007).

The aim of this study was to compare the level of soluble CD44 in saliva and serum between patients with oral SCC and healthy controls as a molecular marker.

\section{Materials and Methods}

After approval from Ethics in research Committee of Babol University of Medical Sciences, a total of 20 patients with primary OSCC (12 women and 8 men) who referred to Shahid Rajaee Hospital in Babolsar (Mazandaran province, north of Iran) who had histological confirmation before starting treatment that don't get any treatment including surgery, radiotherapy or chemotherapy were selected. Twenty healthy subjects of the control group (11 women and 9 men), were selected from patients of Babol dental school, after clinical examination and mucosal health confirmation, according to the age and sex of selected patients. People who had systemic disease or received medicine for systemic disease at the time of study were excluded.
After obtaining oral consent from the subjects, they were first asked to swallow and then put the saliva in a deionized and sterilized plastic tube for 5 minutes without swallow. Saliva were collected under resting conditions in a quiet room between 10.00 AM and 12.00 AM. Subjects should not eat or drink or smoke 90 minutes before sampling. One milliliter of blood was taken from subjects to evaluate serum soluble CD44 levels. The samples of saliva and blood were collected from each group of patients and control was placed on ice after sampling until the laboratory. Then blood and saliva were centrifuged at 2,000 and 11,000 rpm respectively for 10 minutes, and their supernatants were kept in separated micro tubes at $-80^{\circ} \mathrm{C}$ freezer. After completion of sampling to determine the level of soluble CD44, after defrosting the samples at room temperature, they were evaluated by an ELISA test kit (Human sCD44std Platinum ELISA, eBioscience, Vienna, Austria) according to the instructions and, finally, the absorbance of light measured by micro ELISA reader and after calculations, the results were analyzed by SPSS version 22 software between two groups. To compare the means of number and age between women and men who participated in the study and in the case and control groups, chi-square and AVOVA tests were used respectively, and to compare the means of soluble CD44 levels in serum and saliva between the case and control groups T-test was used. Also, Spearman correlation test was used to determine the correlation between means of CD44 levels of serum and saliva in each case and control group. The significance level was considered $\mathrm{P}<0.05$.

\section{Results}

The mean and standard deviation of age of the subjects in patient and control groups were $62.35 \pm 14.27$ and $60.65 \pm 13.33$ years, respectively. Forty percent of the subjects were in patient group and $45 \%$ in the control group were male. The age and sex of the subjects were similar in both patient and control groups and there was no statistically significant difference between the two groups ( $\mathrm{P}$ value $=0.696$ and $\mathrm{P}$ value $=0.5$ respectively $)$. Also, there was no statistically significant difference between mean of age of women and men in the patient group

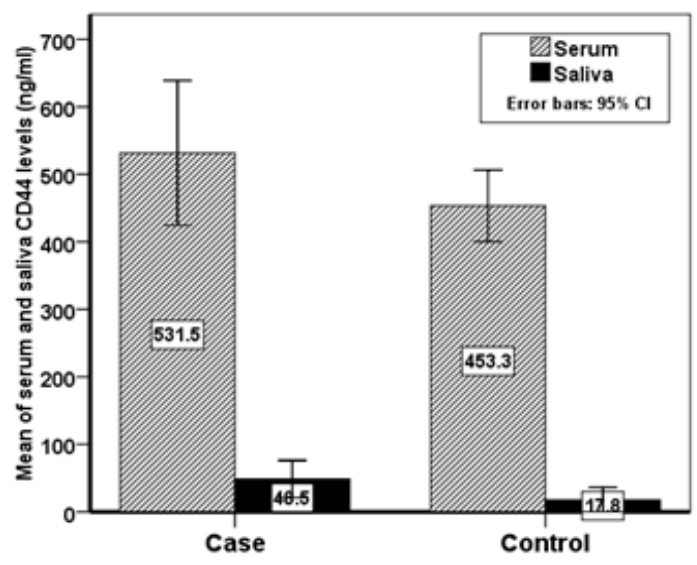

Figure 1. Comparison of solCD44 Protein Level in Serum and Saliva of Patients and Controls 
Table 1. Mean and Standard Deviation of solCD44 Level in Serum and Saliva in Patients and Control Group

\begin{tabular}{llcc}
\hline CD44 & Group & Mean \pm SD $(\mathrm{ng} / \mathrm{ml})$ & P value \\
\hline Serum & Case & $531.51 \pm 228.95$ & 0.182 \\
& Control & $453.3 \pm 113.74$ & \\
\multirow{2}{*}{ Saliva } & Case & $48.53 \pm 59.02$ & 0.061 \\
& Control & $17.76 \pm 39.14$ & \\
\hline
\end{tabular}

$(\mathrm{P}$ value $=0.897)$ and control group $(\mathrm{p}$ value $=0.711)$. In the statistical analysis of the number of women and men there was no significant difference in each of the patient and control groups between them $(\mathrm{P}$ value $=0.371$ and $P$ value $=0.655)$, respectively.

The mean and standard deviation of soluble CD44 level in serum and saliva of the patient and control groups are presented in Table 1. There was no statistically significant difference in serum solCD44 level between the patient and control groups $(\mathrm{P}$ value $=0.182)$. Figure 1 shows the comparison of solCD44 levels of serum and saliva in both patient and control groups. According to the results, there was also no statistically significant difference in saliva solCD44 level between the patient and control groups $(\mathrm{P}$ value $=0.061)$.

Moreover, there was no significant correlation between the solCD44 level in serum and saliva in each patient and control group $(\mathrm{P}$ value $=0.61$ and $\mathrm{P}$ value $=0.445)$.

\section{Discussion}

The clinical findings in this study showed that patients with oral squamous cell carcinoma had an average age of 62 years. This finding is in agreement with most studies and may result from a longer exposure time and cumulative effects to peripheral carcinogens.

In the present study, soluble CD44 level in serum and saliva samples of patients with oral squamous cell carcinoma was compared with healthy subjects. Increased serum solCD44 level was seen in patient group in compare with control group but there was no significant difference; But Khamis et al., in their study, measured all standard isoforms and variants of solCD44, concluded that serum levels of CD44 in patients with OSCC were significantly higher than patients with benign epithelial tumor, as well as healthy subjects in control group (Khamis et al., 2017). High serum levels of CD44 in patients with OSCC could be related to the amount of extracellular contraction of the CD44 antigen released by the remaining stem cells in the tumor mass (Allegra et al., 2012, Patel et al., 2016).

CD44 is known as a poor prognosis marker for detection of SCC cancer stem cells (Khamis et al., 2017). Recent studies indicate that CD44 is an important biomarker for the subset of cancerous stem cells that has the ability to self-regenerate and the capacity to initiation, progression, invasion, metastasis, relapse of tumors, and resistance to chemotherapy and radiotherapy (Koukourakis et al., 2013). This cellular subset was first isolated by Bonnet and Dick, (1997) from acute myeloid leukemia samples. Cancer stem cells, also have been detected from brain tumors, by Singh et al., (2003) and
Salivary and Serum Soluble CD44 Levels in oral SCC Patients

Singh et al., (2003); in prostate tumors by Collins et al., (2005) and Collins et al., (2005); in colorectal cancer by Dalerba et al., (2007); in pancreatic tumors by Li et al., (2007) and in lung tumors by Ho et al., (2007).

In 2007, Prince et al., (2007) recognized a subset of cells in head and neck tumors that indicate surface marker of CD44 with stem cell characteristics. These cells were able to replicate when they were implanted in the body of suppressed immune mice.

Detection of CD44 is important for the study of tumor progression and invasion because invasive tumors attack to extracellular matrix containing tissues for further development; the interaction between CD44 and hyaluronic acid has a decisive role in different cellular pathways (Negi et al., 2012; Nagano and Saya, 2004)

Trapasso and Allegra, (2012) observed a high solCD44 level in patients with laryngeal carcinoma compared with controls. Also, Faber et al., (2011) observed a significant difference in solCD44 level in serum of HNSCC patients compared to control group.

Lin et al., (2010) showed that the serum levels of CD44 in peripheral blood of patients with advanced oral, throat and laryngeal cancer are much higher than healthy subjects, and higher protein levels have inverse relationship with prognosis. Franzmann et al., (2005) found significant difference in solCD44 level between 26 patients with head and neck SCC and 10 healthy subjects. Their results are promising due to the high diagnostic power and the suggestion that solCD44 can be a specific diagnostic marker for head and neck cancer (Franzmann et al., 2007; Allegra et al., 2012; Franzmann et al., 2012). Chen et al., (2014) suggested that detection of solCD44 levels in saliva could be a good predictor in HNSCC diagnosis. Kawano et al., (2005) found a significant difference between mean serum levels of CD44v5, CD44v6, and CD44vst in HNSCC patients with healthy subjects. They found that the mean serum levels of CD44v5, CD44v6 and CD44vst after treatment were significantly lower than their pre-treatment levels, suggesting that solCD44 could be considered a prognostic marker before treatment.

But, as with the results of this study, Franzmann et al., (2005) did not find any significant difference between solCD44 serum levels between oral cancer patients and the control group, which could be due to the relatively small size of oral tumors, which did not have the ability to alter base level of solCD44.

Andratschke et al., (2005) concluded from their research that CD44v6 serum level in initial screening and follow up of HNSCC patients has not clinically significant importance. The results of Van Hal et al., (1999) report that there is no statistically significant difference between the solCD44v6 serum level in HNSCC patients and the control group, which a reason for justifying this finding is measurement of various isoforms of CD44.

Allegra et al., (2012) studied the solCD44 level in saliva of two groups with laryngeal cancer and healthy subjects. The level of this protein in saliva was significantly higher in patients with laryngeal cancer than in the control group. They also reported that this level was higher in the saliva of patients at advanced stages (III and Asian Pacific Journal of Cancer Prevention, Vol $19 \mathbf{3 0 6 1}$ 
IV) than in the early stages of the disease.

Patel et al., (2011) found that there is an increase in the prevalence of OSCC in women than in men due to an increase in the prevalence of smoking and alcohol consumption, HPV, and increased longevity in women. In the present study, women were $60 \%$ of the patients.

Finally, due to the higher serum and salivary levels of solCD44 in OSCC patients compared to healthy subjects, it is suggested that studies be conducted at a wider and larger sample size because it seems to be due to the results of present and other studies, detection of this marker can be a non-invasive, cost-effective, high-specific and sensitive test, like the PSA screening test for prostate cancer and the Pap test for cervical cancer.

With obtained results, it seems that salivary and serum CD44 test holds potential as a screening tool for OSCC. To establish its worth, several objectives must be met:

1) Need to study a larger number of patients for determination the appropriate cutoff point for the salivary and serum CD44 as well as the true sensitivity and specificity

2) Assess the ability to identify early, treatable disease and precancerous lesions

3) Need to control for possible confounding factors including tobacco, alcohol use, and other medical conditions.

In conclusion, determination of solCD44 level in saliva and serum can be a useful method for diagnosis the person's involvement with cancer cells and the cancer in the early stages. But according to the controversial outcomes of past studies, larger and more accurate studies are needed in groups with more cases who are infected with or suspected of being cancer.

\section{Acknowledgements}

The present study is the result of a research project No. 8930223, approved by the Research Council of Babol University of Medical Sciences. The authors would like to thank the Deputy of Research and Technology of Babol University of Medical Sciences for financially supporting the project.

\section{References}

Allegra E, Trapasso S, Sacco A, et al (2012). ELISA detection of salivary levels of $\mathrm{Cd} 44 \mathrm{sol}$ as a diagnostic test for laryngeal carcinomas. J Cancer Sci Ther, 4, 330-4.

Andratschke M, Chaubal S, Pauli C, et al (2005). Soluble CD44v6 is not a sensitive tumor marker in patients with head and neck squamous cell cancer. Anticancer Res, 25, 2821-6.

Bonnet D, Dick JE (1997). Human acute myeloid leukemia is organized as a hierarchy that originates from a primitive hematopoietic cell. Nat Med, 3, 730-7.

Chen J, Zhou J, Lu J, et al (2014). Significance of CD44 expression in head and neck cancer: a systemic review and meta-analysis. BMC Cancer, 14, 15.

Collins AT, Berry PA, Hyde C, Stower MJ, Maitland NJ (2005). Prospective identification of tumorigenic prostate cancer stem cells. Cancer Res, 65, 10946-51.

Dalchau R, Kirkley J, Fabre JW (1980). Monoclonal antibody to a human leukocyte-specific membrane glycoprotein probably homologous to the leukocyte-common (L-C) antigen of the rat. Eur J Immunol, 10, 737-44.

Dalerba P, Dylla SJ, Park IK, et al (2007). Phenotypic characterization of human colorectal cancer stem cells. Proc Natl Acad Sci U S A, 104, 10158-63.

Faber A, Barth C, Hörmann K, et al (2011). CD44 as a stem cell marker in head and neck squamous cell carcinoma. Oncol Rep, 26, 321-6.

Franzmann EJ, Reategui EP, Carraway KL, et al (2005). Salivary soluble CD44: a potential molecular marker for head and neck cancer. Cancer Epidemiol Biomarkers Prev, 14, 735-9.

Franzmann EJ, Reategui EP, Pedroso F, et al (2007). Soluble CD44 is a potential marker for the early detection of head and neck cancer. Cancer Epidemiol Biomarkers Prev, 16, 1348-55.

Franzmann EJ, Reategui EP, Pereira LH, et al (2012). Salivary protein and solCD44 levels as a potential screening tool for early detection of head and neck squamous cell carcinoma. Head Neck, 34, 687-95.

Ghantous Y, Abu Elnaaj I (2017).Global incidence and risk factors of oral cancer. Harefuah, 156, 645-49.

Ho MM, Ng AV, Lam S, Hung JY (2007). Side population in human lung cancer cell lines and tumors is enriched with stem-like cancer cells. Cancer Res, 67, 4827-33.

Khamis AK, Fouad HA, Raslan HS, et al (2017). Diagnostic and prognostic value of cancer stem cell marker CD44 and soluble CD44 in the peripheral Blood of patients with oral Squamous cell carcinoma. Open Sci J, 2, 1-23.

Kawano T, Yanoma S, Nakamura Y, et al (2005). Soluble CD44 standard, CD44 variant 5 and CD44 variant 6 and their relation to staging in head and neck cancer. Acta Otolaryngol, 125, 392-7.

Koukourakis MI, Giatromanolaki A, Sivridis E, et al (2013). High DLL4 expression in tumour-associated vessels predicts for favorable radiotherapy outcome in locally advanced squamous cell head-neck cancer (HNSCC). Angiogenesis, 16, 343-51.

Li C, Heidt DG, Dalerba P, et al (2007). Identification of pancreatic cancer stem cells. Cancer Res, 67, 1030-7.

Lin JT, Chang TH, Chang CS, et al (2010). Prognostic value of pretreatment CD44 mRNA in peripheral blood of patients with locally advanced head and neck cancer. Oral Oncol, 46, e29-33.

Motallebnejad M, Abedi SM, Seyedmajidi M, et al (2014). Evaluation of protective effect of propolis on parotid salivary glands in gamma-irradiated rats. J Contemp Dent Pract, 15, 8-11.

Nagano O, Saya H (2004). Mechanism and biological significance of CD44 cleavage. Cancer Sci, 95, 930-5.

Negi LM, Talegaonkar S, Jaggi M, et al (2012). Role of CD44 in tumour progression and strategies for targeting. J Drug Target, 20, 561-73.

Patel SC, Carpenter WR, Tyree S, et al (2011). Increasing incidence of oral tongue squamous cell carcinoma in young white women, age 18 to 44 years. J Clin Oncol, 29, 1488-94.

Patel S, Shah K, Mirza S, Shah K, Rawal R (2016). Circulating tumor stem like cells in oral squamous cell carcinoma: An unresolved paradox. Oral Oncol, 62, 139-46.

Prince ME, Sivanandan R, Kaczorowski A, et al (2007). Identification of a subpopulation of cells with cancer stem cell properties in head and neck squamous cell carcinoma. Proc Natl Acad Sci U S A, 104, 973-8.

Seyedmajidi M, Shafaee S, Siadati S, et al (2014). Cyclo-oxygenase-2 expression in oral squamous cell carcinoma. J Can Res Ther, 10, 1024-9.

Seyedmajidi M, Sohanian S, Abbaszadeh H, et al (2017). Astrocyte Elevated gene 1 (AEG-1): A promising candidate 
for molecular targeted therapy in oral squamous cell carcinomas. Asian Pac J Cancer Prev, 18, 3301-5.

Seyedmajidi M, Seifi S, Moslemi D, et al (2018). Immunohistochemical expression of TWIST in oral squamous cell carcinoma and its correlation with clinicopathologic factors. J Can Res Ther, 14, 964-9.

Imani R, Seyedmajidi M, Ghasemi N, et al (2018). HLA-G Expression is Associated with an Unfavorable Prognosis of Oral Squamous Cell Carcinoma. Asian Pac J Cancer Prev, 19, 2527-33.

Singh SK, Clarke ID, Terasaki M, et al (2003). Identification of a cancer stem cell in human brain tumors. Cancer Res, 63, 5821-8.

Takamune Y, Ikebe T, Nagano O, et al (2007). ADAM-17 associated with CD44 cleavage and metastasis in oral squamous cell carcinoma. Virchows Arch, 450, 169-77.

Trapasso S, Allegra E (2012). Role of CD44 as a marker of cancer stem cells in head and neck cancer. Biologics, 6, 379-83.

Van Hal NL, Van Dongen GA, Ten Brink CB, et al (1999). Evaluation of soluble CD44v6 as a potential serum marker for head and neck squamous cell carcinoma. Clin Cancer Res, 5, 3534-41.

Wu JY, Yi C, Chung HR, et al (2010). Potential biomarkers in saliva for oral squamous cell carcinoma. Oral Oncol, 46, 226-31.

Yang WC, Chung HR, Wu JY, et al (2010). Potential biomarkers for the cytologic diagnosis of oral squamous cell carcinoma. J Dent Sci, 5, 60-9.

This work is licensed under a Creative Commons AttributionNon Commercial 4.0 International License. 